Pacific Journal of Mathematics

POLYHEDRALITY OF INFINITE DIMENSIONAL CUBES 


\title{
POLYHEDRALITY OF INFINITE DIMENSIONAL CUBES
}

\author{
Thomas E. Armstrong
}

There are several definitions of polyhedrality for infinite dimensional convex sets. We consider each of these in turn and ask whether infinite dimensional cubes are examples. We find that only the concept of polyhedrality put forth by Alfsen and Nordseth admits infinite dimensional cubes as examples. In some sense this concept of polyhedrality is singled out as the only one which properly generalizes the finite dimensional notion of polyhedrality.

1. Cubes. By a cube we shall mean the unit ball of an $M$-space of Kakutani with unit or any affine isomorph. All cubes are affinely equivalent to the unit ball, $\square(X)$, of $\mathscr{C}(X)$ for a suitable compact Hausdorff space $X$ or to $\square^{+}(X)=\square(X) \cap \mathscr{C}^{+}(X)$.

By a compact cube we mean a cube which is compact under some Hausdorff locally convex topology. $\square(X)$ is compact iff $\mathscr{C}(X)$ is a dual Banach space by a theorem of Dixmier [5], [17]. The predual of $\mathscr{C}(X)$ is unique by a theorem of Grothendiek, [21, Theorem 27-4-1]. $X$ must be hyperstonian by another theorem of Dixmier, [6]. Consequently, $X$ is extremally disconnected or Stonian, [6], and the signed normal measures, $\mathcal{N}(X)$, on $X$ must separate $\mathscr{C}(X)$. The unique predual to $\mathscr{C}(X)$ is the $L$-space $\mathcal{N}(X)$ [21, Theorem 27.3.1]. The topology on $\square(X)$ making it compact is the weak topology $\sigma(\mathscr{C}(X), \mathcal{N}(X))$. (In general by $\sigma(S, A)$ we mean the coarsest topology on the set $S$ rendering all functions in $A$ continuous on $S$ ).

An alternative characterization of a compact cube is as the unit ball of $L^{\infty}(S, \Sigma, \mu)$ for a localizable positive measure space. This is because $L^{\infty}$ is an $M$-space with unit which is the dual of $L^{1}(S, \Sigma, \mu)$ because of localizability, [20]. Hence the unit ball of $L^{\infty}$ is a compact cube. Conversely, if $X$ is a compact Hausdorff space with $\square(X)$ a compact cube $\mathcal{N}(X)$ may be represented, via Kakutani's representation theorem for $L$-spaces, as $L^{1}(S, \Sigma, \mu)$ for a localizable positive measure space. In fact $S$ may be taken to be a dense open set in $X, \Sigma$ the locally Borel sets and $\mu$ a positive Radon measure on $S$ built from normal measures on $X$. This was done in essence by Dixmier in [6].

One example of a cube which isn't in general compact is the unit ball of the $B(S, \Sigma)$ of all bounded $\Sigma$-measurable functions on the measurable space $(S, \Sigma) . \quad B(S, \Sigma)$ equipped with the uniform norm is an $M$-space with unit which is $\sigma$-reticulated or Dedekind $\sigma$-complete, [21]. It may 
be represented as $\mathscr{C}(X)$ for a compact Hausdorff $X$. The Dedekind $\sigma$-completeness of $\mathscr{C}(X)$ is equivalent with the property of $X$ known as basic disconnectedness. $X$ is basically disconnected, [9] or $\omega$ extremally disconnected, [21], iff the closure of every Baire open set is open, iff the interior of every Baire compact set is compact.

Every basically disconnected space $X$ is totally disconnected in that it possesses a base of clopen sets. A compact Hausdorff space $X$ is totally disconnected iff it is the Stone space of its Boolean algebra of clopen sets iff $\square(X)$ is the norm closed convex hull of its set, $\xi(\square(X))$, of extreme points. Such cubes will be called Boolean cubes.

2. Polyhedrality. If anything should be an infinite dimensional polyhedron a compact cube should be. We might also expect that a Boolean cube be an infinite dimensional polyhedron under a suitable concept of polyhedrality. We don't believe that the other cubes should be called "polyhedral" since they don't have enough extreme points. We examine the concepts of polyhedrality extant and determine whether or not cubes are examples. We shall be primarily interested in compact cubes.

Phelps, in [18], defines two classes of polyhedra which contain Choquet simplexes. These are the $\alpha$-polytopes and the $\beta$ polytopes. An $\alpha$-polytope is defined as finite codimensional slice of a Choquet simplex. A $\beta$-polytope is defined as an affine image of a Choquet simplex under a map with finite dimensional fibers. Phelps shows that no infinite dimensional centrally symmetric convex compact set is either an $\alpha$-polytope or a $\beta$-polytope. Hence we have the following proposition.

Proposition 1. No infinite dimensional cube is either an $\alpha$-polytope or a $\beta$-polytope.

REMARK. Phelps shows in [18] that the polyhedra of [2] are $\beta$-polytopes.

Before we examine other notions of polyhedrality let us recall, from [1], the notion of a face of a convex set $S$. A convex subset $F$ of $S$ is a face of $S$ iff whenever $\{x, y\} \subset S$ and $\alpha \in(0,1)$ are such that $\alpha x+$ $(1-\alpha) y \in F$ then $\{x, y\} \subset F$. Thus $F$ contains any closed line segment in $S$ for which it contains an interior point. The extreme points, $\xi(S)$, are precisely those $x \in S$ with $\{x\}$ a face of $S$. Both $\phi$ and $S$ are faces of $S$. Faces are preserved under arbitrary intersection and under increasing unions. The faces of $S$ form a complete lattice when ordered by inclusion. There is for any $A \subset S$ a smallest face of $S$, face $(A)$, containing $A$. If $x \in S$, face $(x)$ is defined to be face $(\{x\})$. Any face of a face of $S$ is a face of $S$. If $f$ is an affine function on $S$ the set $\left\{f=\sup _{s} f\right\} \subset S$ is a face of $S$. In particular if $H$ is a supporting 
hyperplane to $S$ in the ambient vector space then $H \cap S$ is a face of $S$ and all faces arise in this fashion. If $S$ is given an affine topology $\tau$ rendering convex combination continuous the $\tau$-closed faces of $S$ are an important object of study. $\tau$-closed faces include $\phi$ and $S$ and are closed under arbitrary intersection. The set of $\tau$-closed faces of $S$ form a complete lattice when ordered by inclusion. If $A \subset S \operatorname{cl}_{\tau}$ face $(A)$ denotes the smallest $\tau$-closed face of $S$ containing $A$. If $x \in A$ let $\operatorname{cl}_{\tau}$ face $(x)=\operatorname{cl}_{\tau}$ face $(\{x\})$. If $\tau$ is $T_{0}$ all elements of $\xi(S)$ give rise to $\tau$-closed faces of $S$. If $f$ is a $\tau$-continuous affine function an $S$ the face $\left\{f=\sup _{s} f\right\}$ is $\tau$-closed but in general not all $\tau$-closed faces of $S$ arise in this manner.

Klee, in [11], defined a convex set $S$ to be polyhedral, or Kleepolyhedral, iff $S \cap M$ is a polyhedron in $M$ for every finite dimensional affine variety $M$ in the ambient vector space. In [16], Lindenstrauss defines a Banach space to be polyhedral iff its ball is Klee-polyhedra. In [16], Lindenstrauss shows that no dual Banach space is polyhedral. Thus, no infinite dimensional compact cube is Klee polyhedral. In [15], Lazar shows that a Lindenstrauss space $E$ (i.e. one whose dual is isometric to an $L$-space) is polyhedral iff it contains no subspace isometric with $\mathscr{C}(N \cup\{\infty\}$ ) (where $N \cup\{\infty\}$ is the one point compactification of the positive integers $N$ ). Furthermore Lazar shows, in [15], that the Lindenstrauss space $E$ is polyhedral iff the unit ball of $E^{\prime}$ has no proper infinite dimensional $\sigma\left(E^{\prime}, E\right)$ closed faces.

Proposition 2. (a) No infinite dimensional cube is Klee polyhedral.

(b) If $X$ is an infinite compact Hausdorf space then $\mathscr{C}(X)$ isn't polyhedral.

(c) A Lindenstrauss space $E$ is polyhedral iff it contains no subspace isometric with $\mathscr{C}(X)$ for any infinite compact Hausdorff space $X$.

(d) If $X$ is an infinite compact Hausdorf space $\mathscr{C}(X)$ has a subspace isometric to $\mathscr{C}(N \cup\{\infty\})$.

Proof. (b). The positive face of the unit ball of $\mathcal{M}(X)=[\mathscr{C}(X)]^{\prime}$ consisting of positive Radon measures on $X$ of norm 1 is known to be $\sigma(M(X), \mathscr{C}(X))$ closed, is infinite dimensional since $X$ is infinite, and is proper. This suffices, in view of Lazar's results, to establish (b).

That (b) implies both (a) and (d) is immediate hence both (a) and (d) are valid.

(c) If a Lindenstrauss space $E$ contains a subspace isometric with $\mathscr{C}(X)$ where $X$ is an infinite compact Hausdorff space it must contain a subspace isometric with $\mathscr{C}(N \cup\{\infty\})$, since $\mathscr{C}(X)$ isn't polyhedral. This establishes one implication of (c). The other implication is immediate.

To proceed further with our examination of polyhedrality of cubes we need to examine the facial structure of cubes. In particular we are 
interested in norm closed faces of cubes, and compact faces of compact cubes. We are interested in faces $F$ which are centrally symmetric in that there is a center $c$ such that the reflection, $2 c-F$, of $F$ in $c$ is equal to $F$. Finally, we are interested in determining face $(x)$ and $\mathrm{cl}_{\tau}$ face $(x)$ where $\tau$ is either the norm topology, $n$, or a compact topology on the cube.

To facilitate the discussion of the faces of a cube it is convenient to use the notion of an order interval in a partially ordered set $(X, \leqq)$. A closed order interval is of the form $[a, b]=\{x \in X: a \leqq x \leqq b\}$ for $\{a, b\} \subset X$ while an open interval is of the form $(a, b)=\{x \in X: a<x<$ $b\}$. By an interval we shall mean a subset $Y$ such that $Y$ is both an increasing and a decreasing family in $X$ and such that if $\{a, b\} \subset Y$ then the closed interval $[a, b]$ is a subset of $Y$. In general open intervals may fail to be intervals only because they aren't increasing or decreasing. If the partially ordered set has the Riesz Interpolation Property so that $x<b$, and $y<b$ implies the existence of a $z$ with $x \leqq z<b$ and $y \leqq z<b$ and $x>a, y>a$ implies the existence of a $z$ with $a<z \leqq x$ and $a<z \leqq y$ then open intervals are intervals. If $X$ is a lattice it has the Riesz Interpolation Property. This is the case for $X=\mathscr{C}(Y)$ with $Y$ a compact Hausdorff space.

Lemma 3. Let $X$ be a compact Hausdorff space and let $\square$ denote the unit ball of $\mathscr{C}(X)$.

(1) If $A \subset B \subset X$ the order interval $\square_{A, B}=\left\{f: \chi_{A}-\chi_{A^{c}} \leqq f \leqq\right.$ $\left.\chi_{B}-\chi_{B^{c}}\right\}$ is a norm closed face of $\square$. All norm closed faces of $\square$ arise in this fashion.

(2) If $F$ is a face of $\square, B=\cup\{\{f=1\}: f \in F\}$, and $A^{c}=$ $\cup\{\{f=-1\}: f \in F\}$ then $A$ is a closed set in the open set $B$ and $\square_{A, B}$ is both the norm closure of $F$ and the smallest norm closed face of containing $F$. If $C$ is a closed subset of the open set $D$ and $\square_{C, D}=\square_{A, B}$ then $C=A$ and $D=B$.

(3) Any face of $\square$ is an order interval.

(4) The closed order interval $[f, g]$ in $\square$ is a face of $\square$ iff $f=$ $\chi_{A}-\chi_{A^{c}}$ and $g=\chi_{B}-\chi_{B^{c}}$ where $A \subset B$ are clopen sets.

(5) The centrally symmetric faces of $\square$ are precisely the faces $\square_{A, B}$ with $A \subset B$ clopen sets.

(6) If $\square$ is compact under a separated affine topology $\tau$ the $\tau$-closed faces are just those faces $\square_{A, B}$ with $A \subset B$ clopen sets.

(7) Let $f \in \square, A=\{f=1\}, B=\{f>-1\}$. Let $n$ denote the norm topology on $\square$ and $\tau$ a separated compact affine topology on $\square$.

(a) $\operatorname{cl}_{n}$ face $(f)=\square_{A, B}$.

(b) $\quad \operatorname{cl}_{\tau}$ face $(f)=\square_{A^{0}, \bar{B}}$.

(8) If $F$ is any face of $\square$ the norm closure of $F$ is a face of $\square$ and, if $\tau$ is a separated compact affine topology on $\square$, the $\tau$-closure of $F$ is a face of $\square$. 
Proof. (3) If $F$ is a face of $\square$ and $\{f, g\} \subset F$ then $\frac{1}{2}[f \vee g+f \wedge g]=$ $\frac{1}{2}[f+g] \in F$. Consequently $\{f \vee g, f \wedge g\} \subset F$. This shows that $F$ is both increasing and decreasing in the order of $\mathscr{C}(X)$. If $f \leqq g$ are in $F$ and $f \leqq h \leqq g$ for some $h \in \square$ the reflection, $(f+g)-h=h^{\prime}$, of $h$ through $\frac{1}{2}[f+g]$ also satisfies $f \leqq h^{\prime} \leqq g$. Consequently $\frac{1}{2}\left[h+h^{\prime}\right]=\frac{1}{2}[f+g] \in F$ so $\left\{h, h^{\prime}\right\} \subset F$. Thus, since $h$ is arbitrary, $F$ contains the entire closed order interval $[f, g]$. This suffices, by the arbitrariness of $f$ and $g$, to establish (3).

(1) That $\square_{A, B}$ is uniformly closed is immediate. Let $f \in \square_{A, B}$ be equal to $\alpha f_{1}+(1-\alpha) f_{2}$ for some $\alpha \in(0,1)$ and $\left\{f_{1}, f_{2}\right\} \subset \square$. We have

$$
\begin{aligned}
f_{1} & =\alpha^{-1} f-(1-\alpha) \alpha^{-1} f_{2} \geqq\left[\alpha^{-1} f-(1-\alpha) \alpha^{-1} f_{2}\right] \vee(-1) \\
& \geqq\left[\alpha^{-1} \chi_{A}-\alpha^{-1} \chi_{A^{c}}-(1-\alpha) \alpha^{-1} \cdot 1\right] \vee(-1) \\
& =\chi_{A}-\chi_{A^{c}} .
\end{aligned}
$$

Similarly $f_{1} \leqq \chi_{B}-\chi_{B^{c}}$ so $f_{1} \in \square_{A, B}$. In a similar fashion we may show that $f_{2} \in \square_{A, B}$. Since, $f, f_{1}, f_{2}, \alpha$ are arbitrary $\square_{A, B}$ is a face of $\square$. This establishes the first assertion of (1). The second assertion of (1) follows from (2).

(2) We first establish the uniqueness assertion. If $\square_{A, B}=\square_{C, D}$ then $\chi_{A}-\chi_{A^{c}} \leqq f \leqq \chi_{B}-\chi_{B^{c}}$ iff $\quad \chi_{C}-\chi_{C^{c}} \leqq f \leqq \chi_{D}-\chi_{D^{c}}$ for $f$ in $\mathscr{C}(X)$. Since $\chi_{A}-\chi_{A^{c}}$ is upper semi continuous and $\chi_{B}-\chi_{B^{c}}$ is lower semi continuous $\chi_{A}-\chi_{A^{c}}$ is the pointwise infimum of $\square_{A, B}$, by known "betweenness" theorems for semi continuous functions on compactspaces. Similarly $\chi_{C}-\chi_{C^{c}}=\inf \left(\square_{C, D}\right)$. This easily implies that $\chi_{\mathrm{A}}-\chi_{\mathrm{A}^{c}}=\chi_{C}-\chi_{C^{c}}$ hence that $A=C$. Similarly $B=D$.

Under the ordering of $\mathscr{C}(X) F$ may be considered as either an increasing net or a decreasing net. We will show that $\inf (F)=\chi_{A}-\chi_{A^{\prime}}$ and that $\sup (F)=\chi_{B}-\chi_{B^{c}}$. It follows from this that $\square_{A \cdot B}$ is the uniform closure of $F$. To see this observe that if $h \in \square_{A, B}$ and $f \leqq g$ are in $F$ then $f \vee(h \wedge g)$ is in $F$. Dini's lemma implies, since $h \wedge g$ is the pointwise infimum of $f \vee(h \wedge g)$ as $f$ decreases in $F$, that $h \wedge g$ is in the closure of $F$ for any $g$ in $F$. Since $h$ is the pointwise supremum of $h \wedge g$ as $g$ increases in $F$ Dini's lemma shows that $h$ is the uniform limit of $h \wedge g$ as $g$ increases in $F$. Thus $h$ is in the uniform closure of $F$ and, since $h$ is arbitrary, $\square_{A, B}$ is in the uniform closure of $F$. Since $F \subset \square$, so is its uniform closure. This establishes our assertion.

To show that $\inf (F)=\chi_{A}-\chi_{A^{c}}$ we show that $A=$ $\cap\{\{f=1\}: f \in F\}$. Since $A^{c}=\cup\{\{f=-1\}: f \in F\}$ this will establish both this statement and the assertion that $A$ is closed. Let $x \in X$ and $f \in F$ with $f(x)<1$. We set $f_{0}=f$ and inductively define a decreasing sequence $\left\{f_{0}, \cdots, f_{n}\right\} \subset F$ which terminates when $f_{n}(x)=-1$. If $\left\{f_{1}, \cdots, f_{k-1}\right\}$ have been defined and $f_{k-1}(x)>-1$ set $f^{k}=f_{k-1}+1-\left|f_{k-1}\right|$ 
and $f_{k}=f_{k-1}-1+\left|f_{k-1}\right|$. We have $1 \geqq f^{k} \geqq f_{k-1} \geqq f_{k} \geqq-1$ and $f_{k-1}=$ $\frac{1}{2}\left[f^{k}+f_{k}\right] \in F$. Thus, since $F$ is a face of $\square,\left\{f^{k}, f_{k}\right\} \subset F$. If $f_{k}(x)>-1$ then $\quad f_{k-1}(x)>0$ and $f_{k-1}(x)-f_{k}(x)=1-f_{k-1}(x)$. Similarly, $f_{j-1}(x)-f_{j}(x)=1-f_{j-1}(x)$ for all $1 \leqq j \leqq k$. We have $1-f_{k}(x)=$ $\left[1-f_{k-1}(x)\right]+\left[f_{k-1}(x)-f_{k}(x)\right]=2\left[1-f_{k-1}(x)\right] . \quad$ By induction we have $2>$ $1-f_{k}(x)=2^{k}\left[1-f_{0}(x)\right]$. Consequently, if $f_{k}(x)>-1$, then $k<$ $\log _{2}\left(2\left[1-f_{0}(x)\right]^{-1}\right)$. This shows that the inductively defined sequence $\left\{f_{0}, \cdots, f_{n}\right\}$ terminates in at most $n=\left[\log _{2}\left(2\left[1-f_{0}(x)\right]^{-1}\right)\right]+1$ steps with $f_{n}(x)=-1$. Thus, we have $x \in A^{c}$ if $f(x)<1$ for some $f \in F$. This is enough to show that $A=\cap\{\{f=1\}: f \in F\}$ hence that $\chi_{A}-\chi_{A^{c}}=$ $\inf (F)$.

In a similar fashion we may show that $B^{c}$ is the closed set $\cap\{\{f=-1\}: f \in F\}$, that $B$ is the open set $\cup\{\{f>-1\}: f \in F\}$, and that $\sup (F)=\chi_{B}-\chi_{B^{c}}$. This establishes (2).

7 (a) is easily established by the same means as we used to establish 2.

(4) If $[f, g]$ is a face it is norm closed. By the proof of $2,[f, g]$ is of the form $\square_{A, B}$ with $\chi_{A}-\chi_{A^{c}}=\inf ([f, g])=f$ and $\chi_{B}-\chi_{B^{c}}=\sup ([f, g])=$ $g$. Thus $\chi_{A}-\chi_{A^{c}}$ and $\chi_{B}-\chi_{B^{c}}$ are continuous, hence $A \subset B$ are clopen sets.

(5) Let $F$ be a symmetric face of $\square$ with center $\gamma$. The norm closure, $\bar{F}$ of $F$ is a centrally symmetric face of $\square$ with center $\gamma$. It is immediate that face $(\gamma)=\bar{F}$ but face $(\gamma) \subset F$ so $F=\bar{F}$. Consequently $F$ is norm closed and of the form $\square_{A, B}$ with $\chi_{A}-\chi_{A^{c}}=\inf (F)$ and $\chi_{B}-\chi_{B^{c}}=\sup (F)$. Since $\quad F=2 \gamma-F \quad \chi_{B}-\chi_{B^{c}}=\sup (2 \gamma-F)=$ $2 \gamma-\inf (F)=2 \gamma-\left(\chi_{A}-\chi_{A^{c}}\right)$. Thus, $\quad 2 \gamma=\chi_{B}-\chi_{B^{c}}+\chi_{A}-\chi_{A^{c}}=$ $2 \chi_{A}-2 \chi_{B^{c}}$. Since $\gamma$ is continuous $A$ and $B^{c}$ are clopen sets.

If $A \subset B$ are clopen sets and $\chi_{A}-\chi_{A^{c}} \leqq f \leqq \chi_{B}-\chi_{B^{c}}$ it is easily seen that the reflection, $f^{\prime}$, of $f$ through $\gamma=\chi_{A}-\chi_{B^{c}}$ also satisfies $\chi_{A}-\chi_{A^{c}} \leqq$ $f^{\prime} \leqq \chi_{B}-\chi_{B^{c}}$. Thus $\square_{A, B}$ has center $\gamma$.

(6) If $\tau$ is a compact Hausdorff affine topology on $\square$ it is of the form $\sigma(\mathscr{C}(X), \mathcal{N}(X))$ where $\mathcal{N}(X)$ is the predual of $\mathscr{C}(X)$. Considering $\mathcal{N}(X)$ as $L^{1}(\mu)$ and $\mathscr{C}(X)$ as $L^{\infty}(\mu)$ for some localizable measure $\mu, \tau$ is of the form $\sigma\left(L^{\infty}(\mu), L^{1}(\mu)\right)$. Any bounded monotone net in $L^{\infty}(\mu)$ is $\sigma\left(L^{\infty}(\mu), L^{1}(\mu)\right)$ convergent. Consequently if $F$ is a $\tau$-compact face of $\square,\{\sup (F), \inf (F)\} \subset F$. When $F$ is a $\tau$-compact face of $\square$ it is a norm closed face of $\square$ hence $\sup (F)=\chi_{B}-\chi_{B^{c}}$ and $\inf (F)=\chi_{A}-\chi_{A^{c}}$ where $A \subset B$. Since $\left\{\chi_{A}-\chi_{A^{c}}, \chi_{B}-\chi_{B^{c}}\right\} \subset f \subset \square, A$ and $B$ are clopen sets.

If $A \subset B$ are clopen sets the face $\square_{A, B}=$ $\left.\left[\left(\chi_{A}-\chi_{A^{c}}\right)+2 \square^{+}\right] \cap\left[\chi_{B}-\chi_{B^{c}}\right)-2 \square^{+}\right]$. Since $\square$ is $\tau$-compact so are $2 \square^{+}$and $\square_{A, B}$.

7 (b) If $\square$ has a compact topology $X$ is extremally disconnected. If $A$ is closed in the open $B$ then $A^{0}$ and $\bar{B}$ are clopen 
sets and $\square_{A^{0}, \bar{B}}$ is a $\tau$-closed face of $\square$ which is the smallest containing $\square_{A, B}=\operatorname{cl}_{n}$ face $(f)$. This shows that $\operatorname{cl}_{\tau}$ face $(f)=\square_{A^{0}, \bar{B}}$.

(8) That the norm closure of any face is a face follows from (2). If $F$ is a face let $\square_{A, B}$ be its norm closure with $A$ closed in the open set $B$. If $\tau$ is a compact topology on $\square$ we must show, after the proof of (7), that $\square_{A^{0}, \bar{B}}$ is the $\tau$-closure of $\square_{A, B}$ which will show that it is the $\tau$-closure of $F$. Now $\tau=\sigma(\mathscr{C}(X), \mathcal{N}(X))$ and all of the elements of $\mathcal{N}(X)$ are normal measures which annihilate sets of first category in $X$. If $\mu \in$ $\mathcal{N}(X)$ then $\mu(\partial A)=\mu\left(A \backslash A^{0}\right)=0=\mu(\bar{B} \backslash B)=\mu(\partial B)$. The increasing net $\square_{A, B}$ has limit $\chi_{B}-\chi_{B^{c}}$. Consequently the limit, for all $\mu$ in $\mathcal{N}(X)$, as $f$ increases in $\square_{A, B}$ of $\int\left(\chi_{\bar{B}}-\chi_{\bar{B}^{c}}\right) \cdot f d \mu$ is zero. Thus $\chi_{\bar{B}}-\chi_{(\bar{B})^{c}} \in \overline{\square_{A, B}}$. Similarly $\chi_{A^{0}}-\chi_{\left(A^{0}\right)^{c}} \in \overline{\square_{A, B}}$. Finally, a similar analysis shows that when $h \in \square_{A^{0}, \bar{B}}$ then $h$ is the $\tau$-limit of $f \vee(h \wedge g)$ as $f$ decreases in $\square_{A, B}$ and $g$ increases in $\square_{A, B}$. Consequently, $\square_{A, B}=\square_{A^{0}, \bar{B}}$ which establishes (8).

REMARKs. (1) If $\square$ is an infinite dimensional cube there are faces which aren't norm closed and norm closed faces which aren't centrally symmetric. The second assertion follows from the existence of open sets in $X$ which aren't closed. The first assertion follows upon the observation that a strictly increasing sequence of open sets $\left\{B_{n}\right\}$ exists in $X$. The faces $F_{n}=\square_{\phi, B_{n}}$ are norm closed and increasing so $\bigcup_{n=1}^{\infty} F_{n}$ is a face. If $F=\bigcup_{n=1}^{\infty} F_{n}$ were norm closed it would be $\square_{\phi, B}$ where $B=\cup_{n=1}^{\infty} B_{n}$. One may find $g_{n} \in F_{n}$ with $g_{n}$ not identically 0 in $B_{n} \backslash B_{n-1}$. The function $g=\sum_{n=1}^{\infty} 2^{-n} g_{n}$ lies in $\bar{F}$ but not in any $F_{n}$.

(2) One criterion for the total disconnectedness of $X$ is that $\square$ be the norm closed convex hull of $\xi(\square)$. Another characterization in terms of faces of $\square$ is that any norm closed face be the norm closure of an increasing union of symmetric faces.

(3) Any centrally symmetric face of a cube is a cube. The compact faces of a compact cube are compact cubes.

We now return to our examination of the polyhedrality of cubes. In [19], Rajagopalan and Roy introduced a generalization of the notion of a $\beta$-polytope. They called the members of this class of convex sets generalized polytopes. We shall call them generalized $\beta$ polytopes. Under their definition a compact convex set is a generalized $\beta$-polytope iff for any point $x$ there is a maximal representing measure, $\mu_{x}$, for $x$ such that, in the convex compact set $\mu_{x}$ of representing measures for $x$, face $\left(\mu_{x}\right)=M_{x}$. Such $\mu_{x}$ are called maximal core representing measures. They show that all $\beta$-polytopes are generalized $\beta$-polytopes and that no infinite dimensional centrally symmetric compact convex set is a generalized $\beta$-polytope. The following proposition follows immediately. 
Proposition 4. No infinite dimensional cube is a generalized $\beta$ polytope.

Lau, in [12], introduces a generalization of the notion of an $\alpha$-polytope. These are called L-polytopes. $\mathrm{Up}$ to affine homeomorphism $L$-polytopes are obtained in the following manner. Take the unit ball $\diamond$ of a dual $L$-space with its weak* topology (Such balls could be called compact octahedra). Select $\left\{h_{1}, \cdots, h_{n}\right\}$ a subset of $A(\diamond)$, the continuous affine functions on $\diamond$. The subset of $\diamond$ given by $\bigcap_{i=1}^{n-}\left\{h_{i}=0\right\}$ is an $L$-polytope. Lau shows that all $\alpha$-polytopes are $L$-polytopes. He also shows that no maximal proper face of an $L$-polytope is centrally symmetric. If $K=\bigcap_{i=1}^{n}\left\{h_{i}=0\right\}$ is an $L$-polytope and $K_{0}$ is a proper closed face of $K$. Lau shows, after Lazar, that there is a proper closed face $\Delta$ of $\diamond$ such that $K_{0}=\Delta \cap K$. Since Lau also shows that all proper closed faces of $\Delta$ are Choquet simplexes, $K_{0}$ is an $\alpha$-polytope. Consequently, from Phelps' result on $\alpha$-polytopes, if $S$ is a compact convex set with an infinite dimensional proper closed centrally symmetric face it isn't an $L$-polytope. These facts make the proof of the following proposition simple.

\section{Proposition 5. No infinite dimensional cube is an L-polytope.}

Proof. We need only consider compact cubes $\square$. Let $X$ be a compact Hausdorff hyperstonian space with $\square$ the unit ball of $\mathscr{C}(X)$ under its compact Hausdorff affine topology. Select $A$ an infinite proper clopen subset of $X$. By (5) and (6) of Lemma 3, the proper closed face $\square_{\phi, A}$ of $\square$ is centrally symmetric hence $\square$ isn't an $L$-polytope.

In [4], Bastiani introduced a notion of polyhedrality for convex sets in a separated locally convex space $(E, \tau)$. Of particular importance was the case when $\tau$ is the finest locally convex topology on $E$. In this case all linear functionals on $E$ are continuous and any face of a convex set is relatively closed. If $S$ is a convex set and $s \in S$ the set cone $(s, S)$ is the smallest convex cone with vertex $s$ containing $S$. If $(E, \tau)$ is separated and locally convex $S$ is Bastiani polyhedral for $\tau$ iff cone $(s, S)$ is $\tau$-closed in $E$ for all $s \in S$. If $\operatorname{cone}(s, S)$ is $\tau$-closed so is its reflection, cone $(s, 2 s-S)$, through $s$. The set cone $(s, S) \cap \operatorname{cone}(s, 2 s-S) \cap S$ is easily verified to be face $(s)$. Consequently if $S$ is $\tau$-closed and Bastiani polyhedral for $\tau$ every face of the form face $(s)$ with $s \in S$ must be $\tau$-closed. We use these facts to establish the following propositions.

Proposition 6. (a) No infinite dimensional cube is Bastiani polyhedral for the norm topology. 
(b) No infinite dimensional compact cube is Bastiani polyhedral for it's compact topology.

Proof. Suppose that the unit ball $\square$ of $\mathscr{C}(X)$ is Bastiani polyhedral for the norm topology. Let $A$ be a compact $G_{\delta}$ in $X$ and let $f \in \square^{+}$ with $\{f=1\}=A$. We have face $(f)=\square_{A, X}$ by 7 (a) of Lemma 1 . If $A$ isn't a clopen set there are infinitely many nonempty $\theta_{n}=\left\{1-2^{-n}<f<\right.$ $\left.1-2^{-n-2}\right\}$. For $\theta_{n}$ empty set $g_{n}=0$. For $\theta_{n}$ nonempty let $g_{n} \in \square^{+}$with $\left\|g_{n}\right\|=2^{-n / 2}$ and with $\operatorname{supp}\left(g_{n}\right) \subset \theta_{n}$. Let $\tilde{f}=f-\sum_{n=1}^{\infty} g_{n} \in \mathscr{C}(X)$. The reader may verify that $1 \geqq \tilde{f} \geqq-1$. Thus $\tilde{f} \in \square$. Since $f \equiv \tilde{f}$ on $A$, $\tilde{f} \geqq \chi_{A}-\chi_{A} c$. Consequently $\tilde{f} \in \square_{A, X}$ so $\tilde{f} \in$ face $(f)$. There is some $\epsilon>0$ with $f+\epsilon(f-\tilde{f}) \in \square$. Consequently $f+\epsilon \cdot \sum_{n=1}^{\infty} g_{n} \leqq 1$ on $X$ for some $\epsilon>0$. In $\theta_{n}$ there is a point $x_{n}$ with $f\left(x_{n}\right)+\epsilon \cdot \sum_{n=1}^{\infty} g_{n}\left(x_{n}\right) \geqq$ $f\left(x_{n}\right)+\epsilon g_{n}\left(x_{n}\right) \geqq\left(1-2^{-n}\right)+\epsilon 2^{-n / 2}$, as long as $\theta_{n} \neq \phi$. Consequently $\epsilon \geqq$ $1-2^{-n}+\epsilon 2^{-n / 2}$ if $\theta_{n}$ is nonempty. For all $n$ with $\theta_{n}$ nonempty $\epsilon \leqq$ $2^{-n / 2}$. Thus, $\theta_{n}$ is empty if $n>-2 \log _{2}(\epsilon)$. Thus $A$ is a clopen set. Since all compact $G_{\delta}$ 's in $X$ are clopen sets $X$ is $\omega$-extremally disconnected. Suppose that $X$ is infinite so there exists a strictly increasing sequence of compact-open sets $\left\{A_{n}: n \in N\right\}$. The union of this sequence is an open $K_{\sigma}$ whose complement is a compact $G_{\delta}$ hence is a clopen set. Thus $\bigcup_{n=1}^{\infty} A_{n}$ is compact. There is a finite integer $m$ with $A_{m}=\cup_{n=1}^{\infty} A_{n}$. This contradicts the strict monotonicity of $\left\{A_{n}: n \in N\right\}$. Consequently, $X$ is finite. Thus, if the unit ball of $\mathscr{C}(X)$ is Bastiani polyhedral for the norm topology $X$ is finite. This is equivalent to assertion (a) of the proposition.

For (b) we note that if $\square$ is an infinite dimensional compact cube with norm topology $n$ and compact topology $\tau$ there is by (a) an $f \in \square$ with face $(f) \subsetneq \mathrm{cl}_{n}$ face $(f) \subset \mathrm{cl}_{\tau}$ face $(f)$.

Alfsen and Nordseth showed, in [3], that the only Choquet simplexes which are Bastiani polyhedral are the finite dimensional ones. Lau, in [12], has established the same result for $L$-polytopes. To render simplexes polyhedral Alfsen and Nordseth weakened Bastiani's condition and only required that for $s$ an extreme point of the convex set $S$ cone $(s, S)$ be closed. This definition was made for $S$ which are compact for some separated locally convex topology $\tau$ on the ambient vector space but may be made for arbitrary $S$. Such a convex set $S$ will be said to be Alfsen-Nordesth polyhedral for $\tau$. Lau, generalizing the result of Alfsen and Nordesth for simplexes, shows in [12] that all $L$-polytopes are Afsen-Nordseth polyhedral. We now establish the same result for cubes.

Proposition 7. (a) All cubes are Alfsen-Nordseth polyhedral for the norm topology. 
(b) All compact cubes are Alfsen-Nordseth polyhedral for their compact topology.

Proof. We let $X$ be compact and Hausdorff. The positive cone $\mathscr{C}^{+}(X)$ is affinely homeomorphic to cone $(e, \square)$ for any $e \in \xi(\square)$ for the norm topology. To see this note that if $e=\chi_{A}-\chi_{A^{c}}$ for some clopen set $A$, then $f \rightarrow e \cdot f+1$ is an affine isometry of $\mathscr{C}(X)$ carrying cone $(e, \square)$ onto $\mathscr{C}^{+}(X)$. If $\mathscr{C}(X)$ is the dual of $\mathcal{N}(X)$ then these maps are $\sigma(\mathscr{C}(X), \mathcal{N}(X))$ homeomorphisms as well. Thus, to establish a) we need only show that $\mathscr{C}^{+}(X)$ is norm closed, which is immediate. To establish (b) we need only show that $\mathscr{C}^{+}(X)$ is $\sigma(\mathscr{C}(X), \mathcal{N}(X))$ closed which is well known.

Concluding remark. We see that the Alfsen-Nordseth criterion for polyhedra is the only one extant which includes all cubes. It would appear to define a universally acceptable class of polyhedra including simplexes, $L$-polytopes and cubes. Dual to the $L$-polytopes we could define a class of $M$-polytopes which are finite codimensional slices of compact cubes. These are easily shown to be Alfsen-Nordseth polyhedra and have in common with the $L$-polytopes only the finite dimensional polyhedra. It would be interesting to determine whether generalized $\beta$-polytopes or Klee polyhedra are Alfsen-Nordseth polyhedral.

\section{REFERENCES}

1. E. M. Alfsen, Convex Compact Sets and Boundary Integrals, Springer-Verlag, BerlinHeidelberg-New York, 1971.

2. - On the geometry of Choquet simplexes, Math. Scand., 15 (1964) 97-110.

3. E. M. Alfsen, and J. Nordseth, Vertices of Choquet simplexes, Math. Scand., 23, (1968), 171-176.

4. A. Bastiani, Cones convexes et pyramides convexe, Ann. Inst. Fourier (Grenoble), 9 (1959), 249-292.

5. J. Dixmier, Sur un Theorem de Banach, Duke Math. J., (1948), 1057-1071.

6. J. Dixmier, Sur Certains Espaces Considérés par M. H. Stone., Sum. Bras. Math., 77 (1951) 151-182.

7. E. G. Effros, Structure in Simplexes, Acta Math., 117 (1967), 103-121.

8. A. J. Ellis, Duality of Partially Ordered Normed Linear Spaces, J. London Math. Soc., 39 (1964), $730-744$.

9. L. Gilman and M. Jerison, Rings of Continuous Functions, Princeton, VanNostrand, 1960.

10. A. Gleit and R. McGuigan, A note on polyhedral Banach spaces, Proc. Amer. Math. Soc., 33 (1972), 398-404.

11. V, Klee, Polyhedral Sections of Convex Bodies, Acta Math., 102 (1959) 243-267.

12. Ka Sing Lau Infinite Dimensional Polytopes, Math. Scand., 32 (1973), 193-213.

13. - The dual ball of a Lindenstrauss space, Math. Scand., 33 (1973), 323-337.

14. A. J. Lazar, The unit ball in conjugate $L_{1}$ spaces. Duke Math. J., 39 (1972), 1-8. 
15. - Polyhedral Banach spaces and extensions of compact operators, Israel J. Math., 7 (1969), 357-364.

16. J. Lindenstrauss, Notes on Klee's paper "polyhedral sections of convex bodies". Israel J. Math., 4 (1966), 235-242.

17. K. F. Ng, On a Theorem of Dixmier. Math. Scand., 29 (1971), 279-280.

18. R. R. Phelps, Infinite dimensional compact convex polytopes, Math. Scand., 24 (1969), 5-26.

19. M. Rajogopalan, and A. K. Roy, Maximal core representing measures and generalized polytopes. Quart J. Math. Oxford, (1974) 257-271.

20 I. Segal, Equivalence of measure spaces, Amer. J. Math., 73 (1951), 257-313.

21. Z. Semadeni, Banach Spaces of Continuous Functions, I, Polish Scientific Publishers, Warsaw, 1971.

Received April 20, 1976. Research supported by National Science Foundation Grant No. MCS 74-05786-A02.

UNIVERSITY OF MINNESOTA

MinNEAPOLIS, MN 55455 



\section{PACIFIC JOURNAL OF MATHEMATICS \\ EDITORS}

RICHARD ARENS (Managing Editor)

University of California

Los Angeles, CA 90024

\section{R. A. Beaumont \\ University of Washington \\ Seattle, WA 98105}

C. C. MOORE

University of California

Berkeley, CA 94720

\section{J. DUGUNDJI}

Department of Mathematics

University of Southern California

Los Angeles, CA 90007

R. FINN AND J. MILGRAM

Stanford University

Stanford, CA 94305

\section{ASSOCIATE EDITORS}

\section{E. F. BECKENBACH}

B. H. NeUmanN

F. WOLF

K. YoSHIDA

\section{SUPPORTING INSTITUTIONS}

\author{
UNIVERSITY OF BRITISH COLUMBIA \\ CALIFORNIA INSTITUTE OF TECHNOLOGY \\ UNIVERSITY OF CALIFORNIA \\ MONTANA STATE UNIVERSITY \\ UNIVERSITY OF NEVADA \\ NEW MEXICO STATE UNIVERSITY \\ OREGON STATE UNIVERSITY \\ UNIVERSITY OF OREGON \\ OSAKA UNIVERSITY
}

\author{
UNIVERSITY OF SOUTHERN CALIFORNIA \\ STANFORD UNIVERSITY \\ UNIVERSITY OF HAWAII \\ UNIVERSITY OF TOKYO \\ UNIVERSITY OF UTAH \\ WASHINGTON STATE UNIVERSITY \\ UNIVERSITY OF WASHINGTON \\ AMERICAN MATHEMATICAL SOCIETY
}

The Supporting Institutions listed above contribute to the cost of publication of this Journal, but they are not owners or publishers and have no responsibility for its contents or policies.

Mathematical papers intended for publication in the Pacific Journal of Mathematics should be in typed form or offset-reproduced (not dittoed), double spaced with large margins. Underline Greek letters in red, German in green, and script in blue. The first paragraph or two must be capable of being used separately as a synopsis of the entire paper. Items of the bibliography should not be cited there unless absolutely necessary, in which case they must be identified by author and Journal, rather than by item number. Manuscripts, in duplicate, may be sent to any one of the four editors. Please classify according to the scheme of Math. Reviews, Index to Vol. 39. All other communications should be addressed to the managing editor, or Elaine Barth, University of California, Los Angeles, California, 90024.

100 reprints are provided free for each article, only if page charges have been substantially paid. Additional copies may be obtained at cost in multiples of 50 .

The Pacific Journal of Mathematics is issued monthly as of January 1966. Regular subscription rate: $\$ 72.00$ a year (6 Vols., 12 issues). Special rate: $\$ 36.00$ a year to individual members of supporting institutions.

Subscriptions, orders for back numbers, and changes of address should be sent to Pacific Journal of Mathematics, 103 Highland Boulevard, Berkeley, California, 94708.

PUBLISHED BY PACIFIC JOURNAL OF MATHEMATICS, A NON-PROFIT CORPORATION

Printed at Jerusalem Academic Press, POB 2390, Jerusalem, Israel.

Copyright (C) 1977 Pacific Journal of Mathematics All Rights Reserved 


\section{Pacific Journal of Mathematics \\ Vol. 70, No. $2 \quad$ October, 1977}

B. Arazi, A generalization of the Chinese remainder theorem ........... 289

Thomas E. Armstrong, Polyhedrality of infinite dimensional cubes .... . . . 297

Yoav Benyamini, Mary Ellen Rudin and Michael L. Wage, Continuous

images of weakly compact subsets of Banach spaces ............ 309

John Thomas Burns, Curvature functions on Lorentz 2-manifolds ......... 325

Dennis F. De Riggi and Nelson Groh Markley, Shear distality and equicontinuity .................................. 337

Claes Fernström, Rational approximation and the growth of analytic

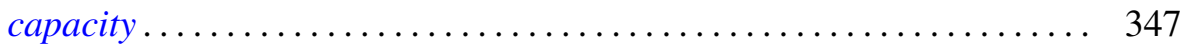

Pál Fischer, On some new generalizations of Shannon's inequality....... 351

Che-Kao Fong, Quasi-affine transforms of subnormal operators ......... 361

Stanley P. Gudder and W. Scruggs, Unbounded representations of

*-algebras........................................ 369

Chen F. King, A note on Drazin inverses .................... 383

Ronald Fred Levy, Countable spaces without points of first countability . . . 391

Eva Lowen-Colebunders, Completeness properties for convergence

spaces ......................................... 401

Calvin Cooper Moore, Square integrable primary representations ....... 413

Stanisław G. Mrówka and Jung-Hsien Tsai, On preservation of

E-compactness ................................ 429

Yoshiomi Nakagami, Essential spectrum $\Gamma(\beta)$ of a dual action on a von

Neumann algebra ................................ 437

L. Alayne Parson, Normal congruence subgroups of the Hecke groups

$G\left(2^{(1 / 2)}\right)$ and $G\left(3^{(1 / 2)}\right)$...

Louis Jackson Ratliff, Jr., On the prime divisors of zero in form rings . . . . 489

Caroline Series, Ergodic actions of product groups .................. 519

Robert O. Stanton, Infinite decomposition bases..................... 549

David A. Stegenga, Sums of invariant subspaces .................. 567 